Published in final edited form as:

Sex Transm Dis. 2014 May ; 41(5): 333-337. doi:10.1097/OLQ.0000000000000116.

\title{
Identifying Syphilis Risk Networks Through Venue Attendance in San Francisco
}

\author{
Sally C. Stephens, MPH ${ }^{\star}$, Charles K. Fann ${ }^{\star}$, Frank V. Strona, MPH ${ }^{*}$, , Wendy Wolf, MPA ${ }^{\star}, \dagger$, \\ Stephanie E. Cohen, MD, MPH ${ }^{\star}$, Susan S. Philip, MD, MPH ${ }^{\star}$, Kyle T. Bernstein, PhD, ScM ${ }^{*}, \neq$ \\ *STD Prevention\&Control Services Section, San Francisco Department of Public Health, San \\ Francisco, CA \\ †Centers for Disease Control and Prevention, Atlanta, GA \\ ‡Division of Epidemiology, Berkeley School of Public Health, University of California, Berkeley, CA
}

\section{Abstract}

Background: Prioritizing interventions for patients with syphilis who are part of large or interconnected sexual networks may be high yield for partner services, and identifying venues named by patients with syphilis who report high numbers of partners may help identify such networks. In this analysis, we explore differences between interviewed patients with early syphilis regarding where they met sex partners.

Methods: With a cross-sectional design, we examined the distribution of total reported sex partners from male index patients with early syphilis interviewed through the San Francisco Department of Public Health partner services program and the self-reported venues named as places they met sex partners. Based on the median number of total partners among male cases of syphilis who named each venue, we categorized venues into 3 levels of partner frequency: high ( $>15$ partners reported), medium (6-15 partners reported), and low ( $<6$ partners reported). Interviewed patients with early syphilis were then classified into these venue categories, and sociodemographic and risk behaviors from electronic medical records and interviews were compared using $\chi^{2}$ tests.

Results: In 2011, 433 male patients with early syphilis named 32 venues. One hundred fortythree (32.3\%) patients were categorized as high, $226(51.0 \%)$ as medium, and $74(16.7 \%)$ as low partner frequency venue users. Patients with early syphilis who reported meeting partners at high partner frequency venues were generally older, more likely to be white, have a previous syphilis infection, use methamphetamines in the previous year, and be HIV infected (all $P<0.05$ ) compared with those who reported meeting partners at medium-frequency and low-frequency venues.

Conclusions: Venues where partners are met may be an appropriate proxy for network membership. Targeting additional resources, outreach, and services to clients who attend highfrequency venues may have a positive impact on syphilis prevention efforts.

Correspondence: Sally C. Stephens, MPH, San Francisco Department of Public Health, 1360 Mission St, Suite 401, San Francisco, CA 94103. Sally.Stephens@ sfdph.org.

Conflicts of Interest: None declared. 
Following record low morbidity rates in the 1990s, the United States' Centers for Disease Control and Prevention launched efforts to eliminate syphilis. ${ }^{1}$ Since those efforts, however, syphilis has increased nationally, with urban men who have sex with men (MSM) most affected. ${ }^{2-4}$ Even after a number of novel interventions were initiated in San Francisco, syphilis rates continue to increase, particularly since 2007. 5,6 In 2011, nearly 700 cases of early syphilis (primary, secondary, or early latent stages) were reported to the San Francisco Department of Public Health, of which $88 \%$ were among MSM. ${ }^{6}$

Partner services have been a core component of syphilis control programs since first described by Thomas Parran ${ }^{7}$ in 1937. Syphilis partner services includes the locating, testing, and treating of partners potentially exposed to syphilis to ensure prompt treatment for infected persons and to preventively treat those who are not yet infected to interrupt further transmission. Many local health departments have developed strategies to improve partner services. One such strategy has been embedding disease intervention specialists (DIS) in community health clinics, which has increased the average number of partners located and decreased the time to the first interview with the newly infected index patient. ${ }^{8}$ Other strategies with varying degrees of success include altering interview techniques to improve recall from patients, ${ }^{9}$ Internet partner services,,${ }^{10,11}$ health promotion campaigns to increase testing, ${ }^{12}$ and online partner notification systems, such as InSpot. ${ }^{13}$ However, the large proportion of unnamed sex partners, particularly among MSM, may be limiting the use of partner services in MSM-focused syphilis epidemics.

Network analyses can provide information that supplements traditional surveillance, illustrating structures of sexual networks and partnerships, as well as areas that may be experiencing outbreaks not apparent from incident data. ${ }^{14-16}$ Information on venues where patients with syphilis meet partners can also be used to supplement traditional surveillance and expand upon network analyses. Venues include both physical and virtual locations, as well as events or combinations of all categories. ${ }^{17}$ Previous research in Baltimore found that patients with syphilis often reported sex partner meeting venues outside their census tract of residence but within a particular geographic area. Given these findings, identifying "riskspace" during interviews was added to the syphilis control program to target resources to geographic areas where high-risk behaviors associated with syphilis transmission occurred. 18,19

Here, we explore data from syphilis index patient interviews, specifically the venues where the patients met sex partners, as a potential proxy for network involvement. We categorized venues based on the level of risk reported by interviewed patients with syphilis who named that venue and explored differences between interviewed patients with syphilis based on venue category. This analysis explores alternative ways to approach targeting interventions and outreach to higher-risk MSM. By focusing on venues frequented by MSM index patients with the highest numbers of sex partners, it may be possible to penetrate sexual networks through these core group members, rather than focusing only on the number of times a venue is mentioned during the interviews of the index patients. 


\section{METHODS}

As mandated by Title 17, California Code of Regulations, all reactive serologic syphilis tests and clinical diagnoses of syphilis are required to be reported to the local health authority. In San Francisco, cases of reported early syphilis are investigated by trained field staff. These investigations include an interview of the index patient as well as treatment verification and elicitation of partners who may have been exposed to syphilis. Data acquired through interviews are collected and maintained in a standardized electronic database.

For the purposes of this analysis, we examined all reported early syphilis cases among males of any age, regardless of sexual orientation, in 2011. Early syphilis cases included those classified as primary, secondary, or early latent stages of infection. ${ }^{20}$ Information on sociodemographics was collected from the electronic medical records and interview records, which are maintained in the same system in San Francisco. We also examined the number of self-reported partners in the last year. History of syphilis was not based on patient selfreport, but instead based on review of all syphilis morbidity events reported to the San Francisco Department of Public Health.

Information on locations where patients report meeting sex partners is captured during the index patient interview. In addition, the index patient is asked if they went to the venue specifically to meet sex partners and if they had sex at that location. Venues that were named by at least 5 individual patients with syphilis in 2011 were included in this analysis. Our analysis was designed to examine which venues should be prioritized for outreach. Because outreach at venues only mentioned by a few individual patients with syphilis would likely not be cost-effective, we a priori excluded all venues named less than 5 times. We determined the median number of total partners in the last year (not named partners) among all patients that named each venue and used this to classify venues as either "high," "medium," or "low" partner frequency venues. Venues where the median number of partners reported from index patients who reported meeting partners at this venue was less than 6 were considered low partner frequency, and venues where the median number of partners was greater than 15 were considered high partner frequency. All other venues were categorized as medium partner frequency. Venue category cutoffs were chosen a priori.

Patients were then hierarchically categorized as attending high, medium, or low partner frequency venues. For example, if someone named at least one high partner frequency venue, they were included in the high partner frequency venue category. Patients stating that they met partners only at low partner frequency venues were included in the low partner frequency category. For patients with syphilis who reported meeting partners at more than 1 type of venue category, we classified the patient as attending the highest partner frequency venue of those reported.

The sociodemographics and risk behaviors of index patients with syphilis were compared across the 3 levels of venue partner frequency. $\chi^{2}$ Tests were used to compare categorical variables, and Wilcoxon rank sum tests were used to compare continuous variables. All analyses were done using SAS 9.3 (SAS Institute, Cary, NC). 
This study was considered exempt from human subjects' considerations in accordance with the Code of Federal Regulations, Title 45, because these data were de-identified and were undergoing retrospective analysis for public health evaluation and improvement purposes.

\section{RESULTS}

In 2011, there were 679 cases of early syphilis reported to the San Francisco Department of Public Health, of which 661 (97.3\%) were among men. ${ }^{6}$ Of these reported cases, 551 (83.3\%) were located and interviewed by STD Prevention and Control Staff. These 551 index patients reported meeting partners at 123 different venues. Of the 123 total venues, 32 venues were named by at least 5 patients in 2011; 6 venues were considered low partner frequency, 19 venues were considered medium partner frequency, and 7 venues were considered high partner frequency. The 32 venues included 12 bars, 2 adult themed book stores, 5 sex clubs/parties, 9 Web sites/apps, and 4 "other" venues. Approximately $80 \%$ of interviewed patients $(n=443)$ named at least 1 of the 32 venues as where they met sex partners and were included in this analysis. A total of 108 interviewed patients $(19.6 \%$ of all interviewed index patients) who did not name at least 1 of the 32 venues were excluded from further analysis.

Table 1 describes the sociodemographics and risk behaviors of the 443 index patients with syphilis included in this analysis. One hundred fifteen (26.0\%) were staged as primary, 143 $(32.3 \%)$ were staged as secondary, and $185(41.8 \%)$ were staged as early latent. The average age of patients was 41.7 years (range, 18-78 years). More than two-thirds of patients were known to be HIV infected, and approximately $60 \%$ of patients were white. One-quarter of patients reported using methamphetamines in the previous year, and the median number of sex partners in the prior 12 months was 10 . Those patients not naming 1 of the 32 venues were similar to the overall demographics of the 443 included patients; however, patients not included were less likely to be white (51\% vs. $60 \%$ ), less likely to use methamphetamines in the previous year ( $14.8 \%$ vs. $25 \%)$, and less likely to be a primary case (18\% vs. $27 \%)$.

When we categorized patients based on the highest partner frequency venue where they reported meeting sex partners during the last year, 143 (32.3\%) were categorized as high partner frequency venue users, $226(51.0 \%)$ were categorized as medium partner frequency venue users, and $74(16.7 \%)$ were categorized as low partner frequency-only venue users (Table 1). There was no difference in stage of disease based on venue partner frequency status; however, there were differences in other demographics and behaviors. Index patients with syphilis who reported meeting any partners at a high partner frequency venue were generally older $(P<0.05)$ and more likely to be white $(P<0.0001)$. In addition, persons that reported meeting partners at high partner frequency venues were more likely to have a previous syphilis infection $(P<0.05)$, use methamphetamines in the previous year $(P<$ $0.05)$, and were more likely to be HIV-infected $(P<0.05)$.

Because some venues may be visited for reasons other than meeting sex partners, we also examined whether persons intended to find sex partners at the venues they named. Among all but 2 of the venues named, $100 \%$ of people said they frequented the locations (physical or virtual) for the purpose of meeting sex partners (Table 2). Of the physical venues (e.g., 
not Internet sites), having sex at the stated venue was most common at book stores and, as expected, sex clubs and parties. As shown in Table 2, those venues identified with the highest median number of total sex partners were not the same as the venues mentioned most frequently by index patients. For example, Sex Club/Party A was only mentioned by 6 index patients, but represents a venue that is frequented by persons with many sex partners. Furthermore, Book Stores A and B were only mentioned by 6 and 11 index patients, respectively, but may represent venues that should be prioritized for outreach and intervention because the index patients who frequent these sites report larger numbers of partners.

Persons were classified based on the highest partner frequency venue named. Within the high partner frequency venue category, most men also reported attending medium and low partner frequency venues. In fact, only one-quarter of high partner frequency venue users exclusively named high partner frequency venues. Men who only named high-risk venues (n = 35) were most likely to be early latent cases $(51.4 \%)$, be white (54.3\%), be HIV infected $(85.3 \%)$, report methamphetamine use (23.5\%), and have a history of syphilis (48.6\%); $20 \%$ were 55 years or older, with a median age of 46.2 years (mean, 45 years). Of those classified as medium partner frequency venue users, $47(20.8 \%)$ reported medium and low partner frequency venues (Figure 1). In addition, there was little difference in the distribution in the number of partners between high and medium partner frequency venues at the 5th and 95th percentile (Table 3). Of the men reporting at least 1 high partner frequency venue, 15 had fewer than 6 partners in the previous year.

\section{DISCUSSION}

The ideal data to examine sexual networks are derived from costly, difficult diary studies. At the programmatic level, resources are limited. We explored an approach that capitalized on data routinely collected through syphilis partner services interviews in San Francisco. We attempted to use data from partner services interviews to identify venues frequented by patients who may be members of a densely connected (or high-transmission) network. However, because the impact of missing data is unknown, we attempted to find data that could be used as a proxy of network relations. Network analyses can be informative in understanding transmission patterns in a geographic region that may not be apparent through traditional surveillance. ${ }^{16} \mathrm{We}$ found that men attending high partner frequency venues were more likely to have a prior syphilis infection and report using methamphetamines in the previous year. In addition, the high partner frequency venues may represent a mixing ground for men who engage in frequent high-risk behavior and those that are only occasional risk takers.

Identifying venues where men with overlapping risk profiles interact may provide an opportunity where both structural and individual interventions can occur. ${ }^{21}$ Men who frequent high partner frequency venues can be identified in index patient interviews and provided with more intensive behavioral counseling and/or partner elicitation. These men may also benefit from enhanced disease control activities, such as more frequent testing, reminders, or being incentivized to test. Likewise, reaching out to venue owners may be an avenue for disease interventions. ${ }^{22-24}$ One recent article suggested that Web site owners may 
be willing to engage in prevention activities that would be both beneficial to and accepted by users. ${ }^{25}$ In San Francisco, these relationships have also extended to sex venue owners and private sex party hosts who actively engage with the health department. In addition to engagement with the health department, venue owners may also implement their own structural interventions that do not rely on public health resources but may still minimize transmission of disease. For example, in San Francisco, party hosts maintain guests list and e-mail all attendees if a partygoer is diagnosed with syphilis; venue owners have also made changes at their own expense to their spaces to provide better space for San Francisco Department of Public Health testing events, have increased lighting throughout spaces, and have created their own educational materials with consultation from the health department (J. McCright Taylor, personal communication, San Francisco Department of Public Health).

Because public health programs must continue to work under strained resources, targeting interventions toward patrons of high partner frequency venues may be a cost-effective way to identify high-transmission networks in a timelier manner than a formal network analysis. Although some venues, such as sex parties/clubs, were not surprising as locations of high partner frequency activities, other physical locations were not as obvious and may warrant more outreach from staff to provide educational materials, easy condom availability and access, and onsite testing events for patrons. Continued monitoring of named venues and categorization of venue partner frequency may provide an additional tool for DIS in partner elicitation and patient management.

In addition, we focused our analysis on venues frequented by MSM index patients with the most sex partners, a possible core group in our local epidemics of HIV and syphilis. These venues, either real or virtual, may represent locations where the core group mixed with lower-risk partners. None of the venues examined were exclusively reported by only highfrequency MSM; therefore, the venues that most of the core group attended may be productive targets for outreach and intervention. We are exploring ways to operationalize this approach with our outreach and partners services programs. Future evaluations will explore targeting venues for outreach and screening that were identified by examining the median number of partners of index patients compared with the those venues that are just mentioned most frequently.

There are several limitations to this analysis. First, data on venues are from patient interviews and may not be representative of all men in San Francisco with syphilis. However, more than $83 \%$ of reported patients with syphilis were successfully interviewed. Furthermore, men who named venues that were not included were excluded from analysis, which may have biased our sample. However, these groups were similar, except for less reported methamphetamine use, less likely to be a primary case, and less likely to be white. Our data also do not reflect all venues used by MSM to find sex partners, and these data do not illustrate where a person may have acquired infection. Similarly, the high partner frequency venues may change rapidly; for example, some venues that were named during 2011 are no longer operating. Individual-level interventions, such as testing reminders or incentives, may be viable options for men who report meeting partners at high partner frequency venues. Lastly, high partner frequency venues may differ between HIV-positive 
and HIV-negative patients. However, we were unable to assess this rigorously given the small sample sizes in the HIV-stratified venue-specific data.

In San Francisco, data on venues where patients with syphilis report meeting sex partners have driven outreach and health promotion activities. Monthly, data on venues named by syphilis and HIV index patients are reported to health promotion staff. Venues that are frequently and consistently named are targeted for outreach materials (posters, palm cards, and condoms), as well as communitybased screening events for syphilis, HIV, chlamydia, and gonorrhea. We propose that venue data may also have value in identifying individuals for targeted, enhanced intervention. One approach may involve more regular screening and active DIS follow-up for cases of syphilis who report meeting partners in high partner frequency venues. Furthermore, more intensive outreach and more frequent screening events at these venues may also be of value. In conclusion, syphilis venue data may help supplement information from network analyses. In addition, these types of analyses can help identify venues that are mixing grounds between men with varying levels of sexual risk behaviors. Using these findings, programs can design both individual and venue based interventions to reduce the syphilis burden within a jurisdiction.

\section{Acknowledgments}

This work was funded in part by the Comprehensive STD Prevention Projects (1H25PS001354-01) Centers for Disease Control and Prevention.

\section{REFERENCES}

1. St Louis ME, Wasserheit JN. Elimination of syphilis in the United States. Science 1998; 281:353354. [PubMed: 9705711]

2. Williams LA, Klausner JD, Whittington WL, et al. Elimination and reintroduction of primary and secondary syphilis. Am J Public Health 1999; 89:1093-1097. [PubMed: 10394323]

3. Kerani RP, Handsfield HH, Stenger MS, et al. Rising rates of syphilis in the era of syphilis elimination. Sex Transm Dis 2007; 34:154-161. [PubMed: 17179773]

4. Centers for Disease Control and Prevention. Sexually Transmitted Disease Surveillance 2011. Atlanta: U.S. Department of Health and Human Services, 2012.

5. Bernstein KT, Stephens SC, Strona FV, et al. Epidemiologic characteristics of an ongoing syphilis epidemic among men who have sex with men, San Francisco. Sex Transm Dis 2013; 40:11-17. [PubMed: 23254114]

6. STD Control Section. San Francisco Sexually Transmitted Disease Annual Summary, 2011. San Francisco, CA: San Francisco Department of Public Health, 2012.

7. Parran T Shadowon the Land: Syphilis. New York: Reynal \& Hitchcock, 1937.

8. Taylor MM, Mickey T, Winscott M, et al. Improving partner services by embedding disease intervention specialists in HIV-clinics. Sex Transm Dis 2010; 37:767-770. [PubMed: 20693936]

9. Brewer DD, Potterat JJ, Muth SQ, et al. Randomized trial of supplementary interviewing techniques to enhance recall of sexual partners in contact interviews. Sex Transm Dis 2005; 32:189-193. [PubMed: 15729158]

10. Ehlman DC, Jackson M, Saenz G, et al. Evaluation of an innovative Internet-based partner notification program for early syphilis case management, Washington, DC, January 2007-June 2008. Sex Transm Dis 2010; 37:478-485. [PubMed: 20539261]

11. Mimiaga MJ, Tetu AM, Gortmaker S, et al. HIV and STD status among MSM and attitudes about Internet partner notification for STD exposure. Sex Transm Dis 2008; 35:111-116. [PubMed: 18007274] 
12. Ahrens K, Kent CK, Montoya JA, et al. Healthy penis: San Francisco's social marketing campaign to increase syphilis testing among gay and bisexual men. PLoS Med 2006; 3:e474. [PubMed: 17194182]

13. Levine D, Woodruff AJ, Mocello AR, et al. in SPOT: The first online STD partner notification system using electronic postcards. PLoS Med 2008; 5:e213. [PubMed: 18942887]

14. Woodhouse DE, Rothenberg RB, Potterat JJ, et al. Mapping a social network of heterosexuals at high risk for HIV infection. AIDS 1994; 8:1331-1336. [PubMed: 7802989]

15. Potterat JJ, Dukes RL, Rothenberg RB. Disease transmission by heterosexual men with gonorrhea: An empiric estimate. Sex Transm Dis 1987; 14:107-110. [PubMed: 3616851]

16. Doherty IA, Serre ML, Gesink D, et al. Sexual networks, surveillance, and geographical space during syphilis outbreaks in rural North Carolina. Epidemiology 2012; 23:845-851. [PubMed: 23007041]

17. Frost SD. Using sexual affiliation networks to describe the sexual structure of a population. Sex Transm Infect 2007; 83(suppl 1):i37-i42. [PubMed: 17664363]

18. Michaud JM, Ellen J, Johnson SM, et al. Responding to a community outbreak of syphilis by targeting sex partner meeting location: An example of a risk-space intervention. Sex Transm Dis 2003; 30:533-538. [PubMed: 12838079]

19. Michaud JM, Johnson SM, Ellen J. Comparison of sex partner meeting venues and residences of syphilis cases in Baltimore. Sex Transm Dis 2004; 31:239-242. [PubMed: 15028939]

20. Centers for Disease Control and Prevention. Case definitions for infectious conditions under public health surveillance. MMWR Recomm Rep 1997; 46:1-55.

21. Wohlfeiler D, Kerndt P. Personal risk and public impact: balancing individual rights with STD and HIV prevention In: Aral S, Fenton K, Lipshutz J, eds. The New Public Health and STD/HIV Prevention: Personal, Public and Health Systems Approaches. New York: Springer Science + Business Media, LLC, 2013.

22. Shilts R. And the Band Played On: Politics, People and the AIDS Epidemic. New York: St Martin's Press, 1987.

23. Wohlfeiler DStructural and environmental HIV prevention for gay and bisexual men. AIDS 2000; 14(suppl 1):S52-S56. [PubMed: 10981475]

24. Woods WJ, Binson D, Pollack LM, et al. Public policy regulating private and public space in gay bathhouses. J Acquir Immune Defic Syndr 2003; 32:417-423. [PubMed: 12640200]

25. Wohlfeiler D, Hecht J, Volk J, et al. How can we improve online HIV and STD prevention for men who have sex with men? Perspectives of hook-up Web site owners, Web site users, and HIV/STD directors. AIDS Behav 2003; 17:3024-3033. 


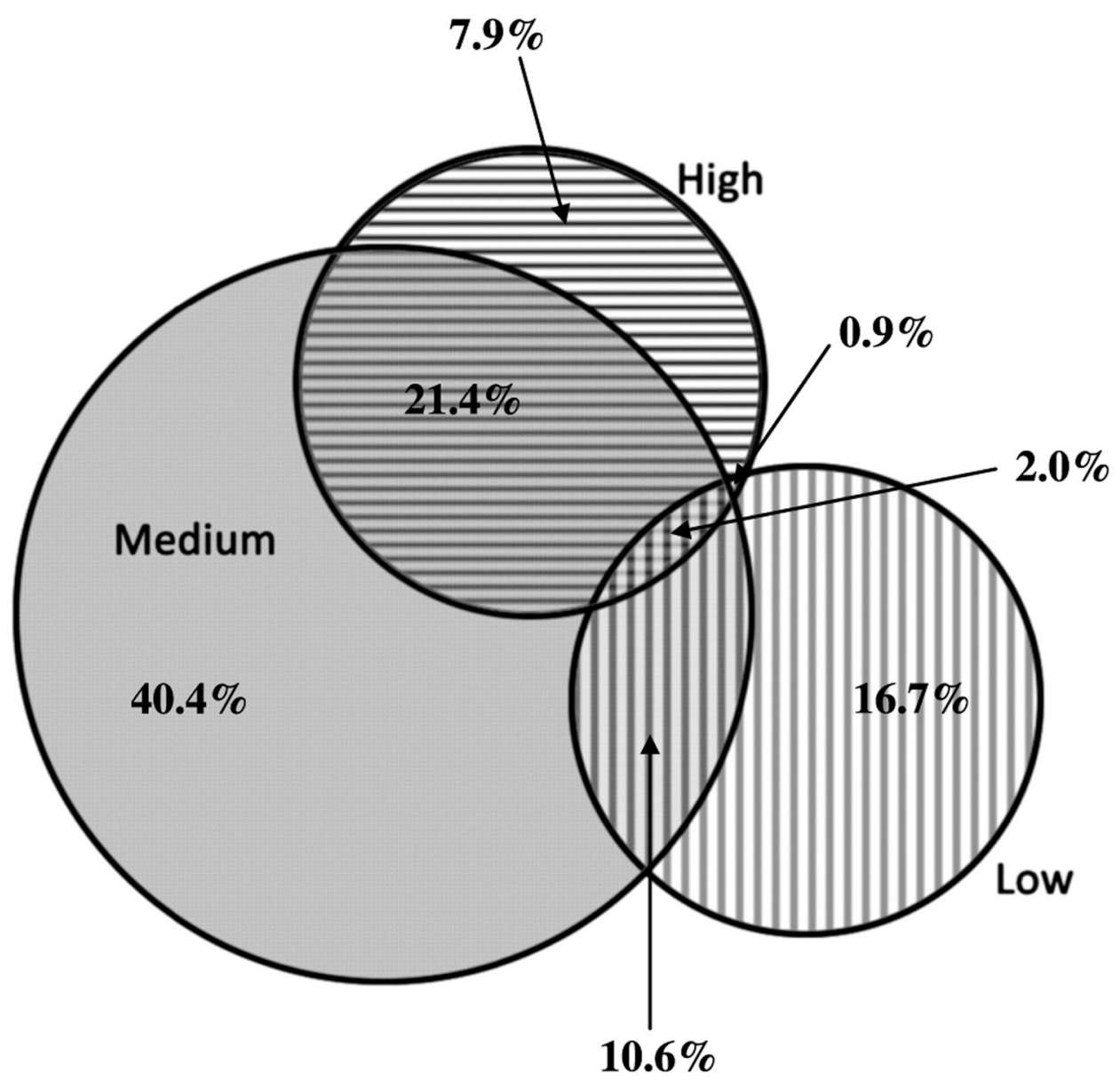

Figure 1.

Distribution of Venue Partner Frequency Among Select Patients With Early Syphilis, San Francisco, 2011 


\section{을 \\ 길}

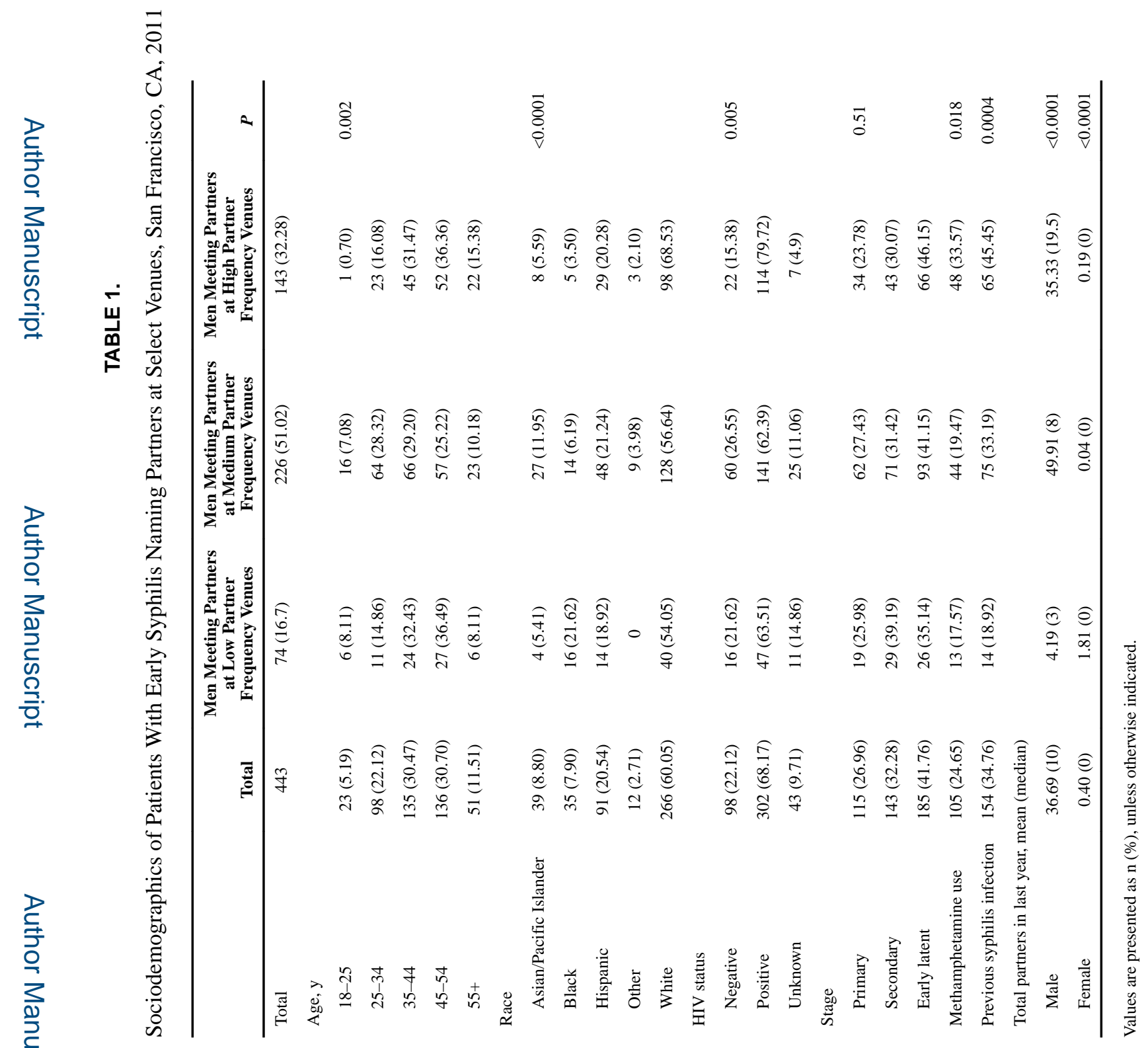

Sex Transm Dis. Author manuscript; available in PMC 2019 November 05. 
TABLE 2.

Median Number of Partners and Frequency of Named Venue

\begin{tabular}{|c|c|c|c|}
\hline Venue Name & $\begin{array}{c}\text { Median No. } \\
\text { Partners } \\
\text { Among Men } \\
\text { Naming Venue }\end{array}$ & $\begin{array}{c}\text { No. Times } \\
\text { Named by } \\
\text { Index Patients }\end{array}$ & $\begin{array}{c}\text { Did You } \\
\text { Have Sex } \\
\text { There?, \% }\end{array}$ \\
\hline Sex Club/Party A & 59 & 6 & 100 \\
\hline Book Store A & 27.5 & 6 & 100 \\
\hline Web site A & 23.63 & 8 & 0 \\
\hline Book Store B & 22.5 & 11 & 90.91 \\
\hline Sex Club/Party B & 21 & 27 & 96.3 \\
\hline Web site B & 20 & 83 & 0 \\
\hline Web site $\mathrm{C}$ & 20 & 6 & 0 \\
\hline Web site D & 17.5 & 44 & 0 \\
\hline Sex Club/Party C & 15 & 19 & 100 \\
\hline Bar A & 15 & 29 & 79.31 \\
\hline Sex Club/Party D & 15 & 60 & 100 \\
\hline Bar B & 12.5 & 7 & 0 \\
\hline Bar C & 12.5 & 5 & 0 \\
\hline Web site $\mathrm{E}$ & 12 & 53 & 0 \\
\hline Web site F & 12 & 42 & 0 \\
\hline Bar D & 10 & 25 & 0 \\
\hline Web site $G$ & 10 & 180 & 0 \\
\hline Bar E & 10 & 7 & 0 \\
\hline Other A & 10 & 13 & 23.08 \\
\hline Sex Club/Party E & 10 & 7 & 85.71 \\
\hline Bar F & 8 & 29 & 0 \\
\hline Web site $\mathrm{H}$ & 8 & 46 & 0 \\
\hline Bar G & 8 & 13 & 0 \\
\hline Other B & 6 & 10 & 100 \\
\hline Bar $\mathrm{H}$ & 6 & 5 & 0 \\
\hline Bar I & 6 & 5 & 0 \\
\hline Bar J & 5.5 & 8 & 0 \\
\hline Bar K & 5.5 & 11 & 0 \\
\hline Bar L & 5 & 9 & 0 \\
\hline Web site I & 4 & 5 & 0 \\
\hline Other $\mathrm{C}$ & 4 & 77 & 0 \\
\hline Other D & 4 & 52 & 54.9 \\
\hline
\end{tabular}

Sex Transm Dis. Author manuscript; available in PMC 2019 November 05. 
TABLE 3.

Number of Partners in the Last Year by Venue Partner Frequency, San Francisco, 2011

\begin{tabular}{lrrccr}
\hline & $\mathbf{n}$ & Mean & Median & $\mathbf{5 \%}$ & $\mathbf{9 5 \%}$ \\
\hline Low & 74 & 4.19 & 3 & 0 & 17 \\
Medium & 226 & 49.91 & 8 & 2 & 100 \\
High & 143 & 35.33 & 19.5 & 3 & 100 \\
\hline
\end{tabular}

